\title{
Pagdalumat sa kalagayang pedagohikal sa akademikong Filipino sa transpormatibong kadawyan
}

Gelilio, Eric $\bowtie$

Sorsogon State University, Philippines (gelilioeric@gmail.com.ph)

Gallanosa National High School, Philippines (eric.gelilio@deped.gov.ph)

Janer, Susan

Sorsogon State University, Philippines (sihjaner@sorsu.edu.ph)

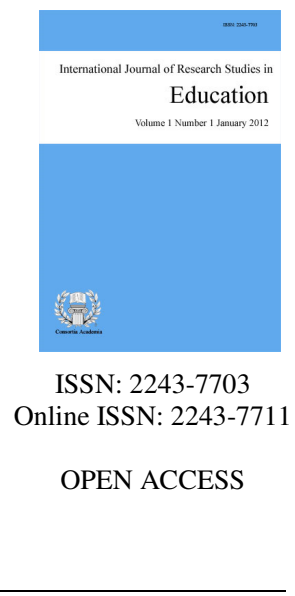

$\begin{array}{ll}\text { Received: } 7 \text { December } 2021 & \text { Revised: } 13 \text { December } 2021 \\ \text { Available Online: } 31 \text { December 2021 } & \text { DOI: } 10.5861 / \text { ijrse.2021.a140 }\end{array}$

Accepted: 31 December 2021

\section{Abstract}

The study was conceptualized to assess the impacts and factors affecting the performance of teachers teaching Filipino discipline in the Division of Sorsogon Province particular in Secondary level of Education, School year 2020-2021 amid of Pandemic. Research utilized descriptive analysis. Purposive sampling was used to gather the data from 1,252 respondents in 22 Secondary schools in Sorsogon Province division. Frequency count, percentage, ranking and chi- square test of independence was employed to analyze the impacts, factors of performance of teachers teaching Filipino subject. Based on analysis of data, teachers of Filipino converged on the higher order of comprehension through their innovations and strategies in teaching language and literature. According to Sandoval (2019), new developed teaching strategy is imperative to ensure the learning progress. Hence, it is important that teachers recreate and enhanced their teaching strategies for the lessons to be enticing for their students. Based on the study results, lack of knowledge in formatting innovations, lack of teaching materials and seminars to learning new teaching techniques are the main contributors and there is a significant connection to the impacts and factors affecting the performance teaching in Filipino. As per Fungo (2015) although teachers already went through excessive trainings, it does not mean that they do not have ineffective habits in teaching language and literature. 3P'S program: Pagtanggap at Pagkilala sa kakayahang Pang-akademik ng mga gurong Filipino was recommended to recognize the innovations, approaches, and teaching methodologies of teachers in different fields of discipline and Gender and Development program and platforms.

Keywords: teaching pedagogy, strategies, innovative strategies, impacts, new normal amid of pandemic 


\section{Pagdalumat sa kalagayang pedagohikal sa akademikong Filipino sa transpormatibong kadawyan}

\section{Introduksyon}

Alinsunod sa RA 4670 o Magna Carta for Public Teachers, Sek. 2, ang guro ay ang mga indibidwal na saklaw ang pagtuturo sa isang silid-aralan, sa iba't ibang antas ng pagtuturo, sa isang buong kasanayan, maging isang guidance counselor, school librarian, pambokasyunal at industriyal na tagasanay, at iba pang indibidwal na sinasaklaw ang pang-administratibo at superbisyon na pinagtibay ng isang pampublikong kagawaran. Hindi na sakop nito ang mga sumusunod: school nurse, school physician, school dentists at iba pang mga manggagawa.

Hindi lingid sa kaalaman ng lahat ang tanging gampanin ng isang guro sa isang dimensyunal na pagtuturo. Saklaw nito ang iba't ibang larangan ng pagkatuto upang higit na malinang ang kakayahan ng bawat mag-aaral sa aspekto ng pagharap sa mga kasalukuyang kaganapan sa lipunan. Ang guro ay kumakatawan sa isang masusing pagsusuri ng mga kaalaman, pagkatuto, abilidad, kakayahan maging pagkatao ng isang indibidwal.

Ang guro bilang isang instrumento ng pagkatuto ay may malawak na responsibilidad ayon sa kanilang sinumpaang katungkulan. Ang guro ay may isang mataas na kredibilidad sa mata ng mga mag-aaral. Sila ang nagsisilbing pundasyon ng sistematiko at organisadong pagbuo ng mag-aaral sa makabuluhang kaisipan, kaugalian at pagkatao.

Sa patuloy na pagbabago ng sistema ng lipunan, maraming mga salik ang higit na nakaaapekto at nakaaambag sa paglinang ng kakayahan ng mga mag-aaral sa iba't ibang larang ng pagtuklas maging sa wika man o panitikan. Mga istilo, pamamaraan, metodo at iba pang teknikal na suporta na naglalayon ng pag-unlad ng kakayahang dimensyunal ng pagkatuto ng isang mag-aaral. Kung higit na susuriin ang tanging layunin ng Kagawaran ng Edukasyon ay lubusang nakatuon sa isang pagkatuto ng mag-aaral, hindi gaanong binibigyan ang pagkakakilanlan ng isang guro sa isang mapamaraan nitong pagkakakilanlan bilang isang natatanging guro sa isang oryentasyong sekswal.

Ayon sa aklat na "Ang Tradisyunal at Makabagong Paraan ng Pagtuturo" (2010), upang maging mabisa at mabilis ang pagkatuto kinakailangan ang paggamit ng mabubuting pamamaraan ng pagtuturo upang mas higit na maging kawili-wili at mabisa ang pagkatuto ng mga mag- aaral. Pinatutunayan na ang pedagohiya sa pagtuturo ay mahalaga upang magkaroon ng kakintalan sa isipan ng mga mag- aaral ang tanging tunguhin ng pag- aaral.

Ayon sa lathala ni Maria Merlisa V. Manuel (2002) na pinamagatang "Guro: Tagahubog ng Kinabukasan ng Sambayanan”, malaki ang impluwensiya ng mga guro sa buhay ng kaniyang mga mag-aaral. Dahil dito, nararapat na pahalagahan ng guro ang kaniyang propesyon hindi lamang bilang isang paraan ng pagkukunan ng pinansiyal na pangangailangan bagkus isang misyon na makapagbibigay ng inspirasyon at pagbabago sa buhay ng isang nilalang Isinasaad dito ang malaking gampanin ng guro sa paghubog ng iba't ibang dimensyunal na pagkatuto ng mga mag- aaral upang higit na maunawaan ang sistema maging pagkakakilanlan sa paksang tinatalakay. Ito ay higit na nagpatibay sa pag- aaral sapagkat binibigyang diin nito ang madiing impluwensiya ng guro sa kaniyang mag- aaral.

Ayon nga kay Pagkalinawan (2015), hindi sapat na maituro ng isang guro kung ano ang paksang - aralin kundi kung paano niya ito maituturo at matututuhan ng mga mag- aaral. Kaya nga, hindi nasusukat ang kahusayan ng guro sa dami ng kaniyang naituturo kundi sa kalidad ng natututuhan ng kaniyang mga estudyante, pinatotohanan na ang guro ay kinakailangang hindi lamang nakatuon sa kung ano ang nalalaman bagkus sa mga makabagong pamamaraan upang higit na malinang ang kakayahan ng mga mag- aaral.

Sa patuloy na pakikipag-ugnayan ng mananaliksik sa Kagawan ng Edukasyon partikular sa mga kaguruan sa 
Pamprobinsiyang Sangay ng Sorsogon, malaki ang hamong dala ng bagong kadawyan sa pagtuturo akademikong Filipino sa iba't ibang dimensyunal na modalidad upang magkaroon ng higit na kakintalan ang karunungan tungo sa layunin ng pagkatuto. Malaki ang nakabalikat sa mga gurong gampanin lalong- lalo na sa pandemyang patuloy na kinahaharap ng lipunan, hindi lang ito sa sakit ng laman kundi isang sakit rin sa pedagohikal na pagtuturo kung paano higit na mabibigyang tugon ang panteknikal na panangailangan ng mga mag- aaral sa disiplinang Filipino. Ang mga nabanggit na mga kadahilanan ang nag-udyok sa mananaliksik upang isagawa ang pag-aaral hinggil sa pedagohikal na pagtuturo sa akademikong Filipino sa Transpormatibong Kadawyan.

\subsection{Paglalahad ng suliranin}

Layunin sa pag-aaral na ito na matukoy ang mga pedagohikal na pagtuturo sa akademikong Filipino sa Transpormatibong Kadawyan (1) Matukoy ang mga salik na nakakaapekto sa pagtuturo ng mga guro sa makabagong kadawyan at sistema ng Kagawaran ng Edukasyon. (2) Malaman ang mga impak ng pagtuturo sa akademikong Filipino. (3) Masuri ang kabuluhang pagkakatulad at pagkakaiba ng salik at impak ng pagtuturo ng guro sa akademikong Filipino batay sa resultang istadistika ng pag- aaral.

\section{Pamaraang ginamit}

Ginamit ang kwantitabong disenyo ng pananaliksik gamit ang istadistikang Chi- Square Test for independence. Ang mga naging kalahok ay binubuo ng 1252 mula sa 22 Pansekundaryang Paaralan sa Una at Ikalawang Pamprobinsiyang Sangay ng Sorsogon na kinakatawan ng mga Medyor at di- medyor na guro na nagtuturo ng akademikong Filipino, mag- aaral, Puno ng Kagawaran at mga Punong- guro. Gumamit ng talatanungan at tseklist para malaman ang mga mga salik at impak ng pagtuturo ng mga guro sa larangan ng wika at panitikan sa pamamagitan ng google form upang makakalap ng mga datos na kinakailangan. Ang pangangalap ng mga datos ay sa loob ng anim na buwan upang higit na maisakatuparan ang pananaliksik. Nagsagawa ng pakikipanayam sa mga kalahok sa pamamagitan ng paghingi ng pahintulot at pagpapaliwanag sa isinagawang pananaliksik sa pamamagitan ng Video call na pamamaraan Ginamit ang Frequency count at pagrarango upang makuha ang mga salik at impak sa pagtuturo sa Akademikong Filipino, pagkatapos ay kinuha ang una at ikalawang ranggo upang maging kategorya sa makabuluhang pagkakatulad at pagkakaiba ng varyabol na salik at impak gamit ang chi-square test of homogeneity. Purposive Sampling ang ginamit ng mananaliksik sa pagkuha ng 30 Gurong medyor sa Filipino na kalahok bilang target ng pananaliksik.

Gumamit rin ng Random Sampling sa pagpili ng mga kalahok sa bawat Paaralan ayon lamang sa mga target na kalahok. Ito ay ginamit para sa mga mag-aaral antas Junior Hayskul at Senior Hayskul, kapwa-guro maging sa mga Puno ng Kagawaran. Ang mga nakalap na datos ay inalisa, sinuri at binigyan ng interpretasyon batay sa istadistikang resulta ng pag- aaral.

\section{Mga natuklasan}

\subsection{Mga Salik na nakaapekto sa pagtuturo ng Akademikong Filipino ng mga guro sa larangan ng Wika at Panitikan}

Ang mga salik na nakaaapekto sa pagtuturo ng Akademikong Filipino ng mga Guro sa larangan ng Wika ay ang kawalan ng inobatibong pamamaraan ng pagtuturo na may 329 na kalahok ang sumagot na may bahagdang 9.35. Pangalawang salik ang kakulangan ng mga istratehiya at pamamaraang pangwika na may 292 o 8.30 bahagdan ng kalahok ang sumagot at sinundan ng walang sapat na kagamitang pampagkatuto bilang salik ng pagtuturo ng Wika ng mga Guro ayon sa 291 na kalahok na sumagot na may bahagdang 8. 27.

Kinakailangan na ang mga guro ay nabibigyan ng panahon para maitala ang kanilang mga bagong-tuklas upang ipakilala rin sa iba pang disiplina at pagkatuklas. Banggit nga sa pag-aaral ni Kizlik (banggit ni Manallo, 2016), sa paggamit ng teknik sa gawaing pagtuturo, magiging epektibo lamang ito kung nauunawaan ng guro 
Gelilio, E., \& Janer, S.

ang pangunahing panuntunan at mga palagay kung saan at paano iaangkop ang isang istratehiya sa pagtuturo.

Mahalaga ayon sa kaniya na magkaroon ng sapat na kagamitan sa pagtuturo maging mga bagong tuklas na pamamaraan at dulog ng pagkatuto.

\section{Talahanayan 1}

Salik na nakakaapekto sa pagtuturo ng mga guro sa Akademikong Filipino sa Larangan ng Wika

\begin{tabular}{|c|c|c|c|c|c|}
\hline Wika & GM & GD & PK & Kabuoan & Ranggo \\
\hline Kakulangan ng mga istratehiya at pamamaraang pangwika & 30 & 245 & 17 & 292 & 2 \\
\hline Walang sapat na kagamitang pampagkatuto & 29 & 256 & 6 & 291 & 3 \\
\hline $\begin{array}{l}\text { Kakulangan sa panahon upang higit na maunawaan ang wika at } \\
\text { gramatika }\end{array}$ & 26 & 210 & 16 & 252 & 4 \\
\hline $\begin{array}{l}\text { Walang sapat na leksikon upang maging batayang panggramatika } \\
\text { ng mga mag-aaral }\end{array}$ & 27 & 178 & 12 & 217 & 7 \\
\hline Walang inobatibong pamamaraan ng pagtuturo & 21 & 289 & 19 & 329 & 1 \\
\hline Kakulangang panggramatikang kasanayan & 29 & 178 & 11 & 218 & 6 \\
\hline Hindi nakaangkla ang mga gurong nagtuturo sa wika & 21 & 173 & 8 & 202 & 8 \\
\hline Walang sapat na pagsasanay sa mga gurong LGBTQ+ & 30 & 200 & 16 & 246 & 5 \\
\hline $\begin{array}{l}\text { Hindi pagtanggap ng institusyon sa pansariling } \\
\text { pagkakakilanlan ng gurong nabibilang sa ikatlong kasarian }\end{array}$ & 8 & 45 & 2 & 55 & 10 \\
\hline Kakulangan ng mga gurong magtuturo ng Akademikong Filipino & 19 & 100 & 12 & 131 & 9 \\
\hline
\end{tabular}

Pananda: GM- Gurong Medyor, PK-Puno ng Kagawaran GD. - Gurong Di- medyor

Samantala, ang salik na nakaaapekto sa pagtuturo ng Panitikan sa Akademikong Filipino ng mga Guro ay ang hindi sapat na pagsasanay o palihan sa pagtuturo ng Panitikan na may 208 na kalahok ang sumagot o 5.91 ayon sa bahagdang pag-aanalisa. Pangalawang salik ang hindi sapat na oras upang basahin ang mga akdang pampanitikan na may 204 o 5.80 na bahagdan ng kalahok ang sumagot at sinundan ng walang inobatibong pagtuturong naisagawa sa panitikan na may 192 kalahok na sumagot kaakibat ang 5.45 na bahagdan. Binanggit sa pag-aaral ni Veer (2015), ang pag-unlad ng isang bansa ay nakasalalay sa kalidad ng mga guro, at ang mga pagsasanay ay mahalaga para sa mga guro. Ang mga "trained" na guro ay mas maraming magagawa kaysa sa mga "untrained" na guro. Kailangan ng mga guro ang patuloy na pag-unlad sa larangan ng edukasyong upang mapaunlad din ang pagtuturo at pagkatuto sa paaralan. Ayon rin kay Sandoval (2015), ang mga tuklas o imbentong paraan ng pagtuturo ay mahalaga upang higit na mabilis, magaan at epektibo ang pagkatuto. Binanggit rin sa kaniyang pag-aaral na maging inobatibo ang mga guro lalong-lalo na sa pagtuturo ng iba't ibang disiplina at larangan.

\section{Talahanayan 2}

Salik na nakakaapekto sa pagtuturo ng mga guro sa Akademikong Filipino sa Panitikan

\begin{tabular}{|c|c|c|c|c|c|}
\hline Panitikan & GM & GD & PK & Kabuoan & Ranggo \\
\hline Hindi pagkilala sa mga local na akda & 25 & 10 & 89 & 124 & 6 \\
\hline $\begin{array}{l}\text { Hindi sapat na pagsasanay at palihan sa pagtuturo ng } \\
\text { panitikan }\end{array}$ & 30 & 11 & 167 & 208 & 1 \\
\hline $\begin{array}{l}\text { Kakulangan ng oras sa pagbibigay ng katuturan sa mga } \\
\text { salitang tekstwal }\end{array}$ & 26 & 14 & 78 & 118 & 7 \\
\hline Kakulangan ng mga dulog at pamamaraan ng pagtuturo & 30 & 18 & 143 & 191 & 4 \\
\hline Walang inobatibong pagtuturo na naisagawa sa panitikan & 30 & 17 & 145 & 192 & 3 \\
\hline $\begin{array}{l}\text { Hindi malinaw na pagpapakilala sa mga akdang } \\
\text { pampanitikan sa ibang karatig lugar ng Pilipinas }\end{array}$ & 14 & 11 & 78 & 103 & 9 \\
\hline $\begin{array}{l}\text { Apektado ang sariling pagkakakilanlan sa pagtuturong moral } \\
\text { ng panitikan }\end{array}$ & 5 & 7 & 76 & 88 & 10 \\
\hline $\begin{array}{l}\text { Pagsasaayos ng istukturang pampanitikan, haba at panahong } \\
\text { kinabibilangan }\end{array}$ & 24 & 1 & 89 & 114 & 8 \\
\hline Kakulangan ng mga babasahing pampanitikan & 26 & 13 & 123 & 162 & 5 \\
\hline $\begin{array}{l}\text { Hindi sapat na oras upang basahin ang mga akdang } \\
\text { pampanitikan }\end{array}$ & 27 & 7 & 170 & 204 & 2 \\
\hline
\end{tabular}

Pinatutunayan ng resultang ito na kinakailangan na magsagawa ng mga bagong tuklas na pamamaraan 
Pagdalumat sa kalagayang pedagohikal sa akademikong Filipino sa transpormatibong kadawyan

maging istratehiya ng pagtuturo lalong-lalo na sa Wika at Panitikan sapagkat hindi madali ang binabalikat ng isang guro lalong-lalo na sa mga Guro na nagtuturo sa akademikong Filipino.

\subsection{Impak ng pagtuturo ng gurong medyor sa mga mag-aaral sa bagong kadawyan}

Ang Positibong impak ng pagtuturo ng isang guro sa akademikong Filipino ay mabisa ang pamamaraan ng pagtuturo sa paraang kaaya-aya at masaya na sinagutan ng 855 na kalahok o 9.50 na bahagdan. Pangalawa ang madaling maunawaan ng mag-aaral ang paksa na sinagutan ng 832 na kalahok o 9.24 na bahagdan at sinundan ng magiliw ang daloy ng pagtuturo na sinagutan ng 756 na kalahok o 8.40 na bahagdan. Binanggit sa pag-aaral nina Carrington at Skelton (2003), nagkakaroon ng kakintalan sa pag-aaral ang mga mag-aaral kung ang kanilang guro ay may kaugnayan sa kanilang sekswal na oryentasyon. Nabanggit rin sa pag-aaral na ang mga gurong nabibilang rito ay may angking kasangkapan ng kasiyahan at kagiliwan sa pagtuturo ng araling pandisiplina. Pinatutuhanan nito na likas na sa mga gurong medyor sa Filipino ang kakayahang magbigay saya at magpangiti sa oras ng klase maging sa ibang aspekto ng pagkatuto. Pinatutunayan na ang isang indibidwal na nabibilang sa ikatlong kasarian ay may katangi-tanging talento upang higit na mapadali ang anumang kahirapang kinahaharap ng lipunan, kung ipapasok ito sa paraang pagtuturo, ang mga gurong nabibilang dito ay may angking kakayahan upang higit na payabungin at pagyamanin ang pagtuturo sa disiplinang kanilang tinuturuan.

\section{Talahanayan 3}

Positibong Impak ng pagtuturo ng guro sa Akademikong Filipino

\begin{tabular}{|c|c|c|c|c|}
\hline Positibo & JHS & SHS & Kabuoan & Ranggo \\
\hline Magiliw an daloy ng Pagtuturo & 400 & 356 & 756 & 3 \\
\hline $\begin{array}{l}\text { Maenganyo ang mga mag-aaral na makiisa sa talakayan ng } \\
\text { Klase }\end{array}$ & 396 & 267 & 663 & 5 \\
\hline $\begin{array}{l}\text { Mapaunlad ang intelektwal na kakayahan ng mag-aaral sa } \\
\text { Akademikong Filipino }\end{array}$ & 234 & 200 & 434 & 10 \\
\hline Madaling maunawaan ng mag-aaral ang paksa & 434 & 398 & 832 & 2 \\
\hline $\begin{array}{l}\text { Inspirasyon ng mga mag-aaral sa patuloy na } \\
\text { pakikipag-ugnayan sa karunungan ng Akademikong Filipino }\end{array}$ & 345 & 231 & 576 & 6 \\
\hline $\begin{array}{l}\text { Mabisa ang pamamaraan ng pagtuturo sa paraang kaaya-aya } \\
\text { at masaya }\end{array}$ & 432 & 423 & 855 & 1 \\
\hline $\begin{array}{l}\text { Gumagamit ng mga larong pangwika at pampanitikan upang } \\
\text { malinang ang kakayahang pangkarunungan }\end{array}$ & 321 & 234 & 555 & 9 \\
\hline $\begin{array}{l}\text { Gumagawa ng mga Inobatibong pamamaraan ng pagtuturo } \\
\text { ng wika at panitikan }\end{array}$ & 267 & 290 & 557 & 8 \\
\hline $\begin{array}{l}\text { Binibigyang pansin ang kahinaan ng mga mag-aaral sa } \\
\text { Akademikong Filipino }\end{array}$ & 343 & 369 & 712 & 4 \\
\hline $\begin{array}{l}\text { Ipinapasok ang sarili sa karanasang global at modern sa } \\
\text { pagkatuto at paglinang ng karunungan panggramatika sa mga } \\
\text { mag-aaral }\end{array}$ & 300 & 267 & 567 & 7 \\
\hline
\end{tabular}

Samantala, ang negatibong impak ng pagtuturo ng mga guro ay sinagutan ng 627 na kalahok na may bahagdang 6. 97 na walang inobasyong nagagawa sa kasulukuyan ang mga kaguruan sa Filipino. Pangalawang negatibong impak na sinagutan ng 374 na kalahok na may 4.16 na hindi sapat ang kaalaman sa pagtuturo ng balarila at pampanitikan na may 4.16 na bahagdan ng kalahok ang sumagot, sinundan naman ng pagkakaroon ng sekswal na relasyon at ugnayan na sinagutan ng 310 na kalahok na may bahagdang 3.44. Napatunayan nga sa pag-aaral ni Coprado (2006), datapwat dumaraan ng mga pagsasanay ang mga kaguruan sa Filipino, hindi pa rin ito sapat na dahilan upang higit na makilala ang mga inobatibong pedagohikal ng mga kaguruan, nagkakaroon pa rin ng takot sa mga kaguruan kung paano maisasatitik ang mga pamamaraang ito upang magamit at maging lunsaran ng mga istratehiya sa makabagong sistema at kadawyan ng Kagawaran ng Edukasyon. 
Gelilio, E., \& Janer, S.

\section{Talahanayan 4}

Negatibong Impak ng pagtuturo ng guro sa Akademikong Filipino

\begin{tabular}{|c|c|c|c|c|}
\hline Negatibo & JHS & SHS & Kabuoan & Ranggo \\
\hline Naaabuso ang kakayahang intelektwal ng mga mag-aaral & 5 & 45 & 50 & 10 \\
\hline $\begin{array}{l}\text { Tradisyunal ma pamamaraan ng pagtuturo ang ipinapamalas sa loob ng } \\
\text { pangkarunungang senaryo }\end{array}$ & 35 & 156 & 191 & 8 \\
\hline Laging huli kung pumasok sa oras ng klase & 56 & 78 & 134 & 9 \\
\hline $\begin{array}{l}\text { Gumagamit ng mga salitang nakakasakit upang ibaba ang kakayahang } \\
\text { pangnilalaman ng mga mag-aaral }\end{array}$ & 123 & 167 & 290 & 4 \\
\hline $\begin{array}{l}\text { Hinahayaan lamang na laging mag-aaral ang umuulat sa unahan na } \\
\text { walang gabay na paraan maging sa aspekto ng pagtuklas ng pagkatuto }\end{array}$ & 34 & 189 & 223 & 5 \\
\hline $\begin{array}{l}\text { Nagkakaroon ng sekswal na ugnayan sa mag-aaral partikular sa } \\
\text { institusyong tinuturuan }\end{array}$ & 165 & 145 & 310 & 3 \\
\hline $\begin{array}{l}\text { Hindi bukas ang ugnayang pampagtuturo sa mga mag-aaral. } \\
\text { Guro-mag-aaral, mag-aaral-guro }\end{array}$ & 145 & 54 & 199 & 7 \\
\hline Walang inobasyon sa pamamaraan ng pagtuturo & 260 & 367 & 627 & 1 \\
\hline Hindi sapat ang kaalaman sa pagtuturo ng balarila at pampanitikan & 143 & 231 & 374 & 2 \\
\hline $\begin{array}{l}\text { Hindi ipinapasok ang e-learning na istilo upang higit na mabigyang pansin } \\
\text { ng iba't ibang karanasan ng mga mag-aaral sa pagkatuto sa wika at } \\
\text { panitikan }\end{array}$ & 132 & 68 & 200 & 6 \\
\hline
\end{tabular}

Pananda: JHS-Junior Hayskul, SHS-Senior Hayskul.

3.3 Kabuluhang pagkakatulad at pagkakaiba ng salik at impak ng pagtuturo ng mga guro sa akademikong

Filipino

\section{Hipotesis}

$>$ Ho- Mayroong kabuluhang pagkakaiba ang salik at impak ng pagtuturo ng mga guro sa akademikong Filipino

$>\quad$ H1- Mayroon kabuluhang pagkakatulad ang salik at impak ng pagtuturo ng mga guro sa akademikong Filipino

\section{Talahanayan 5}

Mga Salik at Impak sa pagtuturo ng akademikong Filipino batay sa resulta ng pagraranggo

\begin{tabular}{|c|c|c|c|c|c|}
\hline Pagtuturo sa Filipino & JHS & SHS & $\mathrm{SH}$ & M & $\mathrm{DM}$ \\
\hline Walang inobatibong pamamaraan sa pagtuturo & 100 & 134 & 9 & 10 & 17 \\
\hline $\begin{array}{l}\text { Hindi sapat na pagsasanay at palihan sa pagtuturo sa akademikong } \\
\text { Filipino }\end{array}$ & 95 & 120 & 8 & 7 & 18 \\
\hline Mabisa ang pamamaraan ng pagtuturo sa paraang kaaya- aya at masaya & 180 & 97 & 6 & 11 & 12 \\
\hline Madaling maunawaan ng mag- aaral ang paksa & 112 & 88 & 1 & 20 & 18 \\
\hline Hindi sapat ang kaalaman sa pagtuturo ng balarila at pampanitikan & 64 & 112 & 1 & 2 & 10 \\
\hline
\end{tabular}

\section{Talahanayan 6}

Istadistika ng mga datos batay sa Chi- square test

\begin{tabular}{|c|c|c|c|c|c|c|}
\hline \multicolumn{7}{|c|}{ Resulta } \\
\hline & 1salik & 2salik & 3impak & 4impak & 5impak & Kabuoan \\
\hline JHS & 100 (118.83) [2.98] & 95 (109. 14) [1.83] & 180 (134.67) [15.26] & 112 (105.18) [0.44] & $64(83.18)[4.42]$ & 551 \\
\hline SHS & 134 (118.83) [1.94] & $120(109.14)$ [1.08] & 97 (134.67) [10.54] & 88 (105.18) [2.81] & 112 (83.18) [9.99] & 551 \\
\hline $\mathrm{SH}$ & 9 (5.39) [2.42] & 8 (4.95) [1.88] & $6(6.11)[0.00]$ & 1 (4.77) [2.98] & $1(3.77)[2.04]$ & 25 \\
\hline M & $10(10.78)[0.06]$ & 7 (9.90) [0.85] & $11(12.22)[0.12]$ & $20(9.54)$ [11.45] & $2(7.55)[4.08]$ & 50 \\
\hline DM & 17 (16.17) [0.04] & 18 (14.86) [0.67] & 12 (18.33) [2.19] & 18 (14.32) [0.95] & $10(11.32)$ [0.15] & 75 \\
\hline Kabuon & 270 & 248 & 306 & 239 & 189 & 1252 \\
\hline
\end{tabular}

Ang chi- square test of independence ay isinagawa upang makita ang kabuluhang pagkakaiba at pagkakatulad ng salik at impak sa kalagayang pedagohikal na pagtuturo sa akademikong Filipino. Pinakita sa resulta na mayroong kabuluhang pagkakatulad ang salik at impak sa resultang 81.16 na ang p- value ay maliit sa 
0. 00001 na tumutugon sa pagtanggap ng Alternate na Hipotesis na binibigyang katuturan ang kabuluhang pagkakatulad ng mga salik at impak sa pagtuturo ng akademikong Filipino sa larang ng wika at panitikan.

Implikasyon na mayroong epekto ang impak at mga salik sa pagtuturo ng akademikong Filipino sa performans ng mga guro sa pagtuturo tungo sa malawakang pagtuklas ng mga kasanayan sa Filipino. Pinatunayan sa pag-aaral ni Fungo (2015), bagaman ang guro ay dumaan na sa maraming pagsasanay hindi pa rin maalis na may pamamaraang hindi pa rin kawili-wili lalo na sa pagtuturo ng wika at panitikan. Kinakailangan na ang mga guro ay nabibigyan ng panahon para maitala ang kanilang mga bagong-tuklas upang ipakilala rin sa iba pang disiplina at pagkatuklas. Banggit nga sa pag-aaral ni Kizlik (banggit ni Manallo, 2016), sa paggamit ng teknik sa gawaing pagtuturo, magiging epektibo lamang ito kung nauunawaan ng guro ang pangunahing panuntunan at mga palagay kung saan at paano iaangkop ang isang istratehiya sa pagtuturo. Mahalaga ayon sa kaniya na magkaroon ng sapat na kagamitan sa pagtuturo maging mga bagong tuklas na pamamaraan at dulog ng pagkatuto.

\subsection{Interbensyon batay sa resulta ng pag-aaral}

Isang panukalang programang 3P'S: Pagtanggap at Pagkilala sa kakayahang Pang-akademik ng mga guro sa akademikong Filipino upang maging batayan ng Kagawaran ng Edukasyon maging ang ahensiyang nagbibigay pagkilala sa mga pedagohikal na dulog at pamamaraan at isang magasing "dalumatrehiya" na kinapapalooban ng mga inobatibong pamamaraan ng pagtuturo ng mga gurong medyor at di- medyor sa pagtuturo ng wika at panitikan sa akademikong Filipino. Maaaring pagbatayan ang pananaliksik na ito ng mga susunod na mananaliksik upang higit na palawigin ang pag-aaral sa mga gurong nagtuturo sa akademikong Filipino sa Pedagohikal na aspekto sa bagong kadawyan sa transpormatibo nitong tunguhin.

\section{Kongklusyon}

Batay sa natuklasan, nabuo ang sumusunod na kongklusyon:

> May mga salik na nakakaapekto sa pagtuturo ng mga guro sa akademikong Filipino sa larangan ng wika at panitikan sa bagong kadawyan at sistema ng Kagawaran ng Edukasyon ay nakategorya sa 3 antas na lumalaman sa pamamaraan ng pagtuturo, pakikitungo at inobatibong dulog.

> May positibo at negatibong impak ang pagtuturo guro sa akademikong Filipino sa larangan ng wika at panitikan na nakategorya sa 3 antas na lumalaman sa pedagohiya, pakikipag- ugnayan at inobatibong dulog.

> Mayroong kabuluhang pagkakatulad ang epekto ng mga salik at impak sa pagtuturo ng mga kaguruan sa Filipino sa larangan ng wika at panitikan.

$>\quad$ Nabuo ang isang panukalang programang 3p's upang mas maituro pa nang may kahusayan ang wika at panitikan at mapanatili ang mataas na lebel ng performans ng mga gurong nagtuturo sa akademikong Filipino.

\subsection{Rekomendasyon}

Batay sa mga resulta at kongklusyon ng pag-aaral na ito, ang sumusunod na mga rekomendasyon ay nabuo:

> Bigyan ng pansin ang mga guro sa kanilang pagtuturo ng akademikong Filipino at bigyan ng pagkilala ang mga natatanging kakayahan ng mga gurong ito na makapagtuklas ng mag bagong pamamaraan o inobatibo sa pagtuturo ng larangan ng wika at panitikan tulad ng pagkakaroon ng mga salingkurang-pagsasanay sa mga gurong pang- akademik sa Filipino kung paano maisasatitik ang mga inobatibong pamamaraan ng pagtuturo sa larangan ng wika at panitikan. 
Gelilio, E., \& Janer, S.

$>\quad$ Pataasin ang antas ng kaalaman ng mga mag- aaral sa pagtuturo ng akademikong Filipino sa pamamagitan ng pagbubukas sa mga inobatibong pamamaraan ng pagtuturo upang maisakatuparan ang mga kasanayan sa isang aralin sa pamamagitan ng paggamit ng mga pamamaraang angkop, sistematik at higit na matunton ang kompetensi ng pagkatuto.

$>\quad$ Kung maaari ay gamitin ang panukalang 3P'S: Pagtanggap at Pagkilala sa kakayahang Pang-akademik ng mga guro sa Akademikong Filipino upang maging batayan ng Kagawaran ng Edukasyon.

$>\quad$ Maaring pagbatayan ang pananaliksik na ito ng mga susunod na mananaliksik upang higit na palawigin ang pag-aaral sa mga gurong nagtuturo sa akademikong Filipino ayon sa tunguhin ng susunod na mananaliksik.

\section{Talasanggunian}

Aguda, L. A., Tamayo, R., \& Leoncion, B. (2013). Effects off heritage tourism to the municipality of Taal, Batangas, Philippines.

Alamer, R. L. (2015). Effects of discussion-interaction method on the performance of grade IV pupils in Science [Unpublished master's thesis]. Sorsogon State College, Sorsogon City.

Almario, V. S. (2015). KWF Manwal sa Masinop na pagsulat (5th ed.). Komisyon ng Wikang Filipino.

Angara, B. J. (n.d.). House Bill 7165 "Lesbian Gay Rights Act of 1999”.

Arambuyong, J. E. (2018). Istilo sa Pagtuturo ng Balarila at Panitikang Filipino [Unpublished master's thesis]. Sorsogon State College, Sorsogon City.

Bramwell, B., \& Lane, B. (2010). Sustainable tourism and the evolving roles of government planning. Journal of Sustainable Tourism, 18(1), 1-5. https://doi.org/10.1080/09669580903338790

Bucjan, E. R. (n.d.). International Journal of English literature and social sciences.

Espina, L. (2007). Modyul Sa Filipino 1: Komunikasyon Sa akademikong Filipino. Mindshapers Co., Inc.

Gebhard, J. (2006). Teaching English as a foreign or second language, second edition. https://doi.org/10.3998/mpub.147778

House Bill No. 258 “An Act of Prohibiting Discrimination based on Sexual Orientation and Gender Identity and Expression (SOGIE EQUALITY ACT). (n.d.).

Huevos, M. M. (2015). Laro: Isang Estratehiya sa Pagtuturo ng Numeracy [Unpublished master's thesis]. Sorsogon State College, Sorsogon City.

Lucas, R. D. (2008). Mga mabisang Istratehiya sa pagtuturo ng Panitikan sa mga mag-aaral ng Kolehiyo [Unpublished master's thesis]. Bulacan State University, City of Malolos.

Mejia, R. C., Fertijo, B., Borbon, J., \& Barlan, L. (2013). Involvement of the community in promoting marine biodiversity as tourist attraction. Journal of International Academic Research for Multidisciplinary.

Pagkalinawan, L. (2015). Ang tradisyunal at mirabegron paraan ng pagtuturo.

Villanueva, V. M. (n.d.). Wikang Filipino: Wikang panlahat, salalayan sa pagtatamo ng likas-kayang pag-unlad, 1-6.

Zafra, G. (2016). Ang pagtuturo ng wika at kulturang Filipino sa disiplinang Filipino (Konteksto Ng K-12).

Katipunan: Journal ng mga ng mga Pag-aaral sa Wika, Panitikan, Sining at Kulturang Filipino, 1, 4-28. https://doi.org/10.13185/kat2016.00102 